\title{
Quaternary Optical Transmission System Combining Phase and Polarization-Shift Keying
}

\author{
Jaume Comellas, Joan M. Gené, Student Member, IEEE, and Josep Prat, Member, IEEE
}

\begin{abstract}
A new optical transmission scheme which combines polarization-shift keying and differential phase-shift keying on the same optical carrier is proposed. Simulations carried out show a considerable tolerance toward different physical impairments, such as polarization-mode dispersion, nonlinear effects, and chromatic dispersion. When compared to other multilevel modulation formats, it shows the advantage of reusing the carrier power, thus, sensitivity is improved.
\end{abstract}

Index Terms-Chromatic dispersion, differential phase-shift keying (DPSK), polarization-mode dispersion (PMD), polarization modulation.

\section{INTRODUCTION}

$\mathbf{P}$ OLARIZATION-MODE dispersion (PMD) and fiber nonlinear effects are currently considered as the key problems of high bit rate $(>10 \mathrm{~Gb} / \mathrm{s})$ fiber-optic transmission systems. While nonlinear effects are alleviated by using constant power modulation formats [1], PMD is difficult to counteract because of its random nature [2]. A possible way to overcome not only the PMD problem but also the chromatic dispersion is by using multilevel modulation formats. By increasing the symbol duration, dispersion effects will reduce. Different multilevel modulation formats have been proposed recently [3]-[5]. This letter introduces a scheme which combines binary polarization-shift keying (PolSK) [5] and differential phase-shift keying (DPSK) [6] on the same optical carrier. The quaternary format obtained allows us to double the transmission capacity or, alternatively, to double the bit period. Since the proposed format has constant power, it shows a high tolerance to nonlinear effects.

\section{Proposed Scheme}

The block diagram of the proposed transmission system can be seen in Fig. 1. Light is first phase modulated (Data 1, a nonreturn-to-zero (NRZ) stream, which is precoded to achieve DPSK format). At the phase-modulator (PM) output, light is polarization splitted [polarization beam splitter (PBS)] and its horizontal component is phase modulated again (with Data 2, also NRZ). At the polarization beam combiner (PBC) output, the state of polarization (SOP) of the signal will take the linear values $45^{\circ}$ (corresponding to a space on Data 2) or $-45^{\circ}$ (mark on Data 2), assuming that the SOP of the input light is linear with $45^{\circ}$ of azimuth.

Manuscript received November 17, 2003; revised February 24, 2004. This work was supported by Nortel Networks-Harlow Laboratories.

The authors are with the Department Signal Theory and Communications, Universitat Politècnica de Catalunya, 08034 Barcelona, Spain (e-mail: jprat@ tsc.upc.es).

Digital Object Identifier 10.1109/LPT.2004.828422

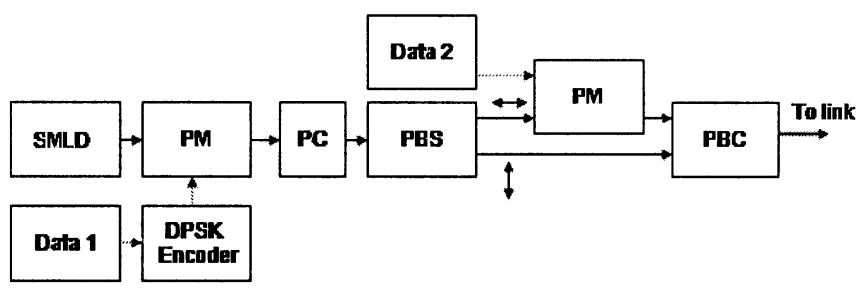

(a)

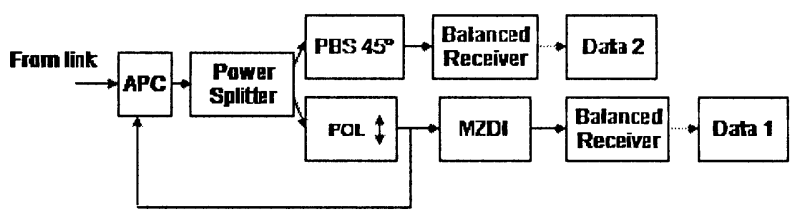

(b)

Fig. 1. Block diagram of the proposed transmission system. SMLD: single-mode laser diode. MZDI: Mach-Zehnder differential interferometer. (a) Transmitter. (b) Receiver.

The polarization controller (PC) placed before the PBS is a key element on the system proposed here. It has to be adjusted in order to obtain a $53^{\circ}$ linear SOP at the PBS input, hence, power is asymmetrically distributed between the two PBS outputs. The lower arm will carry more power than the upper $\operatorname{arm}\left(\sin ^{2} 53^{\circ}\right.$ toward $\cos ^{2} 53^{\circ}$ ). This asymmetry will be very useful to recover the signal at the receiver.

Fig. 1(b) shows the receiver structure. It consists of two independent arms to recover both transmitted signals. After appropriately adjusting the received SOP and splitting the power between both branches, the PolSK receiver is based in a PBS followed by a balanced detector, while the DPSK receiver has a polarizer $[(\mathrm{POL})$ to block the polarization modulated signal], a Mach-Zehnder differential interferometer, and a balanced detector. Low-pass filters and decision stages are placed after the detectors. Due to the POL effect, the input power to the PolSK part is higher than that of the DPSK part (which only uses the vertical linearly polarized component). This implies that the sensitivity of the PolSK receiver would be better if power were equally split between both branches. Thus, by changing the power splitting ratio at the transmitter PBS, the whole system is adjusted in order to have the same sensitivity for both receivers. When the light SOP at the PBS input is set to linear with $53^{\circ}$ of inclination, more power is transferred to the lower arm. On one hand, SOPs at the PBC output will be $53^{\circ}$ and $-53^{\circ}$ (closer to the linear vertical SOP), so the extinction ratio for the PolSK signal will be reduced and its performance will be slightly worse. But on the other hand, the DPSK receiver sensitivity will improve, approaching that of the PolSK receiver. This azimuth value has been theoretically 
obtained by equaling the SNR of both receivers and considering the amplified spontaneous emission (ASE) noise generated at the optical preamplifier as the main limiting factor. Subsequent simulations have shown a good agreement with this $53^{\circ}$ value.

Having more power on the vertical component of the optical field is very useful to correctly recover the signals SOP at the receiver. Assuming that the fiber has produced an unknown SOP transformation to the signal, the automatic polarization controller (APC) placed at the receiver input has to be adjusted to align the input SOP with the polarizing elements of the receiver. The average power detected after the vertical POL placed at the DPSK receiver will be used as the reference signal to govern the APC [bottom arrow in Fig. 1(b)]. By continuously maximizing this power, dynamic polarization tracking is ensured. Taking into account that the APC acts on the whole signal, also, the PolSK signal will have the appropriate SOP.

\section{Simulation Results}

To demonstrate the feasibility of the proposed system, some simulations (using VPI TransmissionMaker) were done. Some parameters holding through the different simulations are as follows:

1) Each channel (PolSK and DPSK) carries $10 \mathrm{~Gb} / \mathrm{s}$, so a $20-\mathrm{Gb} / \mathrm{s}$ signal is transmitted.

2) Simulations have been carried out with 1024 bit streams from a pseudorandom binary sequence (PRBS) $\left(2^{10}-1\right)$ generator.

3) Using a PRBS $\left(2^{10}-1\right)$ generator, each simulation consisted of two 1024 bit streams (one for each channel).

4) The fiber span utilized is $50 \mathrm{~km}$ long and its dispersion and PMD coefficients have been varied during the different simulations. With respect to PMD, only first order, quantified as differential group delay (DGD), has been considered, and the fiber principal axes [principal states of polarization (PSPs)] have been adjusted to check all the possible cases.

5) An optical preamplifier, with a 5-dB noise figure value is present at the receiver input. A variable attenuator is placed just before it. Receiver sensitivity is measured at the optical preamplifier input.

6) In order to reduce the ASE noise, the signal is optically filtered with $50-\mathrm{GHz}$ bandwidth after the preamplifier.

7) By adjusting the laser azimuth to $53^{\circ}$, a sensitivity (for bit-error rate $(\mathrm{BER})=10^{-9}$ ) of $-37 \mathrm{dBm}$ is obtained for both channels in the back-to-back case, limited by the optical preamplifier noise; the Gauss-intersymbol interference method is used to estimate the BER value.

8) At the receiver, a polarization misalignment of $3^{\circ}$ is always considered to simulate a realistic situation. It has to be highlighted that DPSK is much more sensitive to the polarization misalignment than PolSK. When misalignment approaches $10^{\circ}$, DPSK suffers a $3-\mathrm{dB}$ penalty while PolSK rests unaffected.

First, the performance of the system with respect to chromatic dispersion was investigated. The results obtained demonstrated that the DPSK signal is more robust to chromatic dispersion. The DPSK receiver supported $800 \mathrm{ps} / \mathrm{nm}$ with negligible

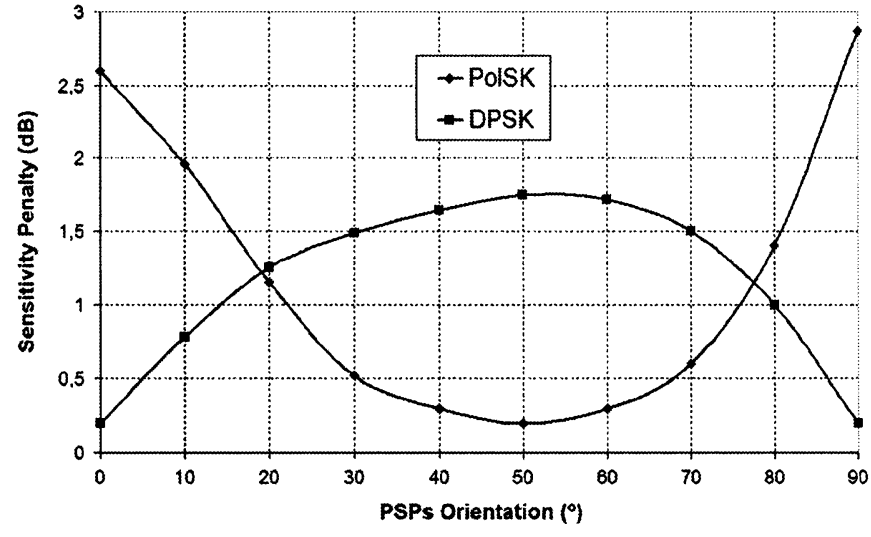

Fig. 2. Sensitivity penalty (with respect to a back-to-back value of $-37 \mathrm{dBm}$ ) versus PSPs orientation when DGD $=20$ ps. Each channel is carrying $10 \mathrm{~Gb} / \mathrm{s}$.

penalty while the PolSK suffered 3-dB penalty with less than $200 \mathrm{ps} / \mathrm{nm}$. This implies that in a practical implementation of the proposed system, a dispersion compensation method should be used. Robustness toward nonlinearities (which is one of the advantages of constant envelope formats such as our proposal) has been assessed by increasing the output transmitter power; negligible penalties have been observed when output power ranges from 0 to $20 \mathrm{dBm}$.

Fiber birefringence was applied in order to generate DGD and evaluate the system performance toward PMD. The fiber PSPs were changed to cover all the possible cases (see Fig. 2). It is important to highlight that by rotating the PSPs of the transmission fiber, very different conditions are imposed to the system. As a simple exemple, when PSPs coincide with the vertical and horizontal axes, the DPSK part will remain unaffected (the DPSK modulated signal is only present at the vertical component of the beam), while the PolSK part will be under very strict conditions (SOP states for the PolSK modulated signal are $53^{\circ}$ and $-53^{\circ}$ ).

It is observed that the DPSK part is highly robust to PMD when the PSPs are $0^{\circ} / 90^{\circ}$ (the $0^{\circ}$ component is eliminated so the signal is unaffected by PMD). In this particular case, the DPSK signal supports DGD $=50$ ps with $<1 \mathrm{~dB}$ of penalty with respect to the back-to-back configuration. On the other hand, when fiber PSPs are near $50^{\circ} / 140^{\circ}$, a worst case is reached for the DPSK stream (a 1.7-dB penalty is obtained with DGD = 20 ps) but an excellent performance is achieved for PolSK (it supports 55 ps of DGD with a penalty $<2 \mathrm{~dB}$ ). The DPSK part performs in general better (the PolSK penalty is unacceptable when PMD $>40 \mathrm{ps}$ and the PSPs are $0^{\circ} / 90^{\circ}$ ).

The DPSK performance is improved when the PolSK modulator is active (turning off the PolSK part, the sensitivity of the DPSK receiver degrades by $1 \mathrm{~dB}$ ). This constitutes an additional advantage of the proposed system. This is explained as by modulating the SOP (applying the PolSK), the erbium-doped fiber amplifier polarization hole burning is avoided in optically amplified systems [7], so a better performance is achieved.

Received eye diagrams when both signals are simultaneously transmitted are shown in Fig. 3. While DPSK rests unaffected by PolSK, the effect of the DPSK modulation on the PolSK signal can be detected as a widening on the falling edges of the pulses. In any case, the eye remains opened enough under the simulated conditions so as to correctly detect the transmitted data. 

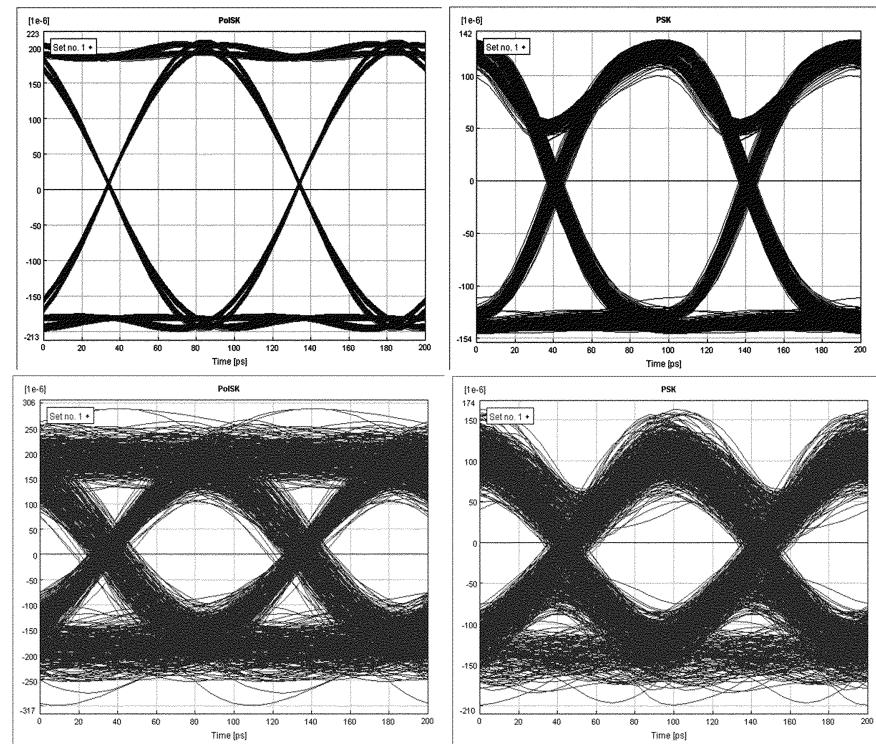

Fig. 3. Received eye diagrams [(a) and (c) PolSK, and (b) and (d) DPSK]. Upper: ideal (without optical preamplifier noise). Lower: with PMD $=40 \mathrm{ps}$ and PSPs oriented at $30^{\circ} / 120^{\circ}$.

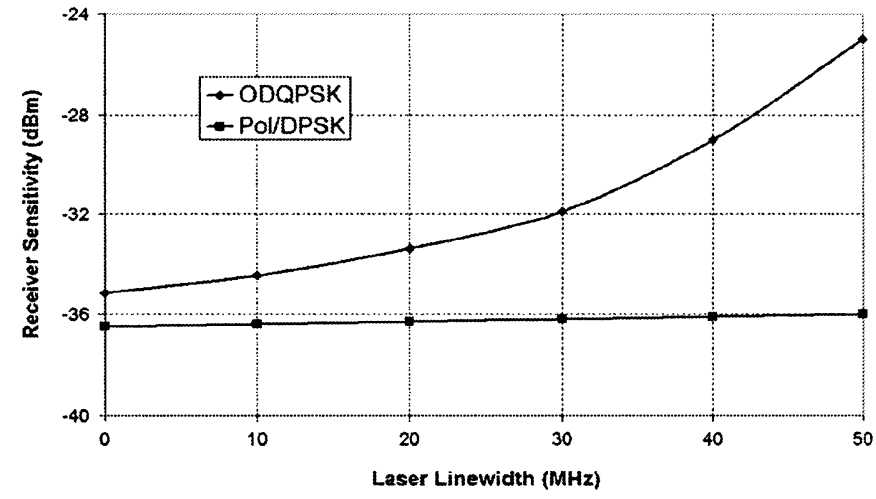

Fig. 4. Sensitivity versus laser linewidth for the PolSK/DPSK and the ODQPSK systems.

In order to compare the performance of the proposed system with other advanced quaternary systems, some simulations were done using an optical differential quadrature phase-shift keying (ODQPSK) modulated system [8]. The ODQPSK format is more robust toward chromatic dispersion and PMD but our proposal is much more tolerant to phase noise. Fig. 4 shows the results obtained in terms of sensitivity versus laser linewidth. When ranging from 0 to $50 \mathrm{MHz}$, the PolSK/DPSK system suffers a penalty of only $0.5 \mathrm{~dB}$. On the other hand, the ODQPSK system suffers high penalties with linewidths higher than $20 \mathrm{MHz}$ (which is a typical specification of single-mode lasers). The greater tolerance of our proposal toward phase noise is due to the fact that in the ODQPSK format, the phase distance between symbols is $90^{\circ}$, half of DPSK (PolSK is even more insensitive to phase noise).

It has also to be highlighted that our system presents $1.5 \mathrm{~dB}$ of sensitivity improvement (with respect to the ODQPSK) for the back-to-back case. This is due to better use of the carrier power. A fraction of the power, that corresponding to the vertical polarization, is modulated twice, so it contributes to both receivers.

\section{DisCUSSION}

A new modulation format combining DPSK and PolSK has been proposed and its performance has been demonstrated by means of simulation. An original method of distributing asymmetrically the power between the polarization axes allows optimizing the system performance. As a fraction of the carrier power is used twice (PolSK is applied over the DPSK modulated signal), a better receiver sensitivity is achieved. Tolerance to chromatic dispersion, fiber nonlinearities, and PMD has been investigated with $2 \times 10 \mathrm{~Gb} / \mathrm{s}$. A possible application could consist of using the system as a highly robust $10-\mathrm{Gb} / \mathrm{s}$ transmission system. By applying the same data to both branches, the original information would be correctly recovered even under very stringent conditions. As there is always a good signal quality in any of the receiver branches, best performing channel should be selected at the receiver.

\section{REFERENCES}

[1] M. Rohde et al., "Robustness of DPSK direct detection transmission format in standard fiber WDM systems," Electron. Lett., vol. 36, no. 17, pp. 1483-1484, Aug. 2000.

[2] H. Sunnerud et al., "Polarization-mode dispersion in high-speed fiber-optic transmission systems," J. Lightwave Technol., vol. 20, pp. 2204-2219, Dec. 2002.

[3] M. Ohm and J. Speidel, "Quaternary optical ASK-DPSK and receivers with direct detection," IEEE Photon. Technol. Lett., vol. 15, pp. 159-161, Jan. 2003.

[4] S. Walklin et al., "Multilevel signalling for increasing the reach of 10 Gb/s lightwave systems," J. Lightwave Technol., vol. 17, pp. 2235-2248, Nov. 1999.

[5] R. Noe, D. Sandel, and F. Wust, "Polarization mode dispersion tolerance of bandwidth-efficient multilevel modulation schemes," in Optical Fiber Communication Conf. 2000, vol. 2, Mar. 2000, pp. 198-200.

[6] H. Kim et al., "Polarization-mode dispersion impairments in direct-detection differential phase-shift-keying systems," Electron. Lett., vol. 38, no. 18, pp. 1047-1048, Aug. 2002.

[7] N. S. Bergano et al., "Bit-synchronous polarization and phase modulation scheme for improving the performance of optical amplifier transmission systems," Electron. Lett., vol. 32, no. 1, pp. 52-54, Jan. 1996.

[8] J. M. Gené et al., "Investigation of $10 \mathrm{~Gb} / \mathrm{s}$ optical DQPSK systems in presence of chromatic dispersion, fiber nonlinearities and phase noise," IEEE Photon. Technol. Lett., vol. 16, pp. 924-926, Mar. 2004. 